DEMONSTRATIO MATHEMATICA

Vol. XVII Noj2 1984

Bronisław Przybylski, Zbigniew Zekanowski

\title{
ON DIFFERENT NOTIONS OF THE LINEAR INDEPENDENCE OF VECTOR FIELDS ON A DIFFERENTIAL SPACE
}

\section{Introduction}

Let $M$ and $I /$ be differential spaces with differential structures $C$ and $D$, respectively. If $I: M \rightarrow N$ is a smooth map, then $X_{f}(M, N)$ denotes the C-module of all smooth veotor fielde on 11 tangent to $\mathbb{N}$ (ses [2]). Por arbitrary vector fields $x_{1}, \ldots, x_{k} \in \mathfrak{X}_{f}(M, N)$, we introduce some different notions of Inear independerice at the pointe of $M$ and consider connections between them. What deserves one s attention is Theorem 3.4 and corollariea following from 1 t.

If $A \subset M$, then $\left.f\right|_{A}$ denotes the restriction of $f$ to A. Moreover, if $\alpha \in C$ and $X \in \dot{X}_{f}(M, N)$, then by $\left.\alpha\right|_{A}$ and $\left.x\right|_{A}$ we mean the restrictions of $\alpha$ and $X$ to $A$, respectively. $C_{A}$ denotes the localization of $C$ to $A$. We observe that $C_{A}$ is the structure of differential subspace $A$ induced from $M$ by inclusion. similarly, if $X$ is an arbitrary submodule of $x_{f}(M, N)$, then by $\mathfrak{X}_{A}$ we mean the localization of $X$ to A. It is esey to see that $\mathfrak{X}_{A}$ is a submodule of the $C_{A}$ - module $\left.\mathfrak{X}_{f}\right|_{A}(A, N)$.

If $\mathbb{M}=\mathbb{N}$ and $P$ is the identity map, then we write $X(M)$ instead of $\mathfrak{x}_{P}(M, N)$.

1. Definitions and examples

De 1 i Ition 1.1. Vector fielde $x_{1}, \ldots, x_{k} \in$ $\in \mathfrak{X}_{f}(M, N)$ are said to be C-1inearly independent at a point 
$p \in x$ if, for any $\alpha^{1}, \ldots, \alpha^{k} \in C$, the oondition $\alpha^{1} x_{1}+\ldots+\alpha^{k} x_{k}=0$ Impliog the equalities $\alpha^{1}(p)=\ldots=\alpha^{-k}(p)=0$.

$\Delta_{C}\left(x_{1}, \ldots, X_{k}\right)$ will denote the set of all polnts $p \in M$ such that the reotor fields $x_{1}, \ldots, x_{k}$ are $C-1 i n e a r l y$ independent at p.

of course, the voctor fields $x_{1}, \ldots, x_{k}$ are C-linearly Independent if and only if $\Delta_{c}\left(x_{1}, \ldots, x_{k}\right)=x$.

D. 1 in 1 t 1 n 1.2. Veotor Plelds $x_{1}, \ldots, x_{k} \epsilon$ $\in X_{f}(M, N)$ are sald to be regularly C-Iinearls 1ndependent at a point $p \in \mathbb{M}$ if there exists an open neighbourhood $V$ of $p$ such that the rector flelde $x_{1 \mid \nabla}, \ldots, x_{k \mid \nabla} \in \mathfrak{X}_{f \mid v}(M, \mathbb{N})$ are $\mathrm{C}_{\mathrm{\gamma}}$-linearly Independent.

$\Delta_{\text {reg-C }}\left(x_{1}, \ldots, x_{k}\right)$ will denote the set of all pointe $p \in M$ such that the vector fielde $x_{1}, \ldots, z_{k}$ are regularly C-linearly Independent at $p$.

D 11 n 1 t 10 1.3. Vector flelde $x_{1}, \ldots, x_{k} \epsilon$ $\in X_{p}(M, N)$ are sald to be linearly independent at a point $p \in M$ if the vectors $x_{1}(p), \ldots, x_{k}(p)$ are 11nearly independent at $p$.

$\Delta\left(x_{1}, \ldots, x_{k}\right)$ will denote the set of all pointe $p \in M$ suoh that the veotor fielde $x_{1}, \ldots, X_{k}$ are linearly independent at p.

As an lluatration of these definitions, let us tare the following examples:

F $x$ a m $p$ i 1.4. Let $B$ be a differential space of real numbers with natural atructure. Consider the vector fleld $X=x \frac{\partial}{\partial x} \in \mathfrak{X}(B)$ and note that $\Delta_{C}(X)=\Delta_{\text {Ieg-C }}(X)=B$ but $\Delta(X)=$ $=E \backslash\{0\}$.

Exa m P I 1.5. Iot $1: B \rightarrow B^{2}$ be the inolusion map. Consider the rector flelds $X_{1}, X_{2} \in X_{1}\left(B, E^{2}\right)$ defined as f0110ws: $x_{1}=\left.\alpha\left(\frac{\partial}{\partial x}+\frac{\partial}{\partial y}\right)\right|_{B}$ and $x_{2}=\left.\left(\frac{\partial}{\partial x}-\frac{\partial}{\partial y}\right)\right|_{B}$, where

$$
\alpha(x)=\left\{\begin{array}{l}
0 \text { for } x \leqslant 0 \\
a^{-\left(\frac{1}{x}+\frac{1}{1-x}\right)} \text { for } 0<x<1 \\
0 \text { for } x \geqslant 1 .
\end{array}\right.
$$


It is oasy to vorify that $\Delta_{C}\left(x_{1}, x_{2}\right)=\{x \in B, 0 \leqslant x \leqslant 1\}$ and $\Delta_{r \otimes B-C}\left(x_{1}, x_{2}\right)=\Delta\left(x_{1}, x_{2}\right)=\{x \in \mathbb{E}: 0<x<1\}$.

$B \times$ i $p 101,6$. Lot $K=\left\{(x, y) \in B^{2}: x y=0\right\}$, and

let $1: K \longrightarrow B^{2}$ be the Inolueton map. Conelder the vootor flolds $x_{1}=\left.x \frac{\partial}{\partial x}\right|_{K}, x_{2}=\left.y \frac{\partial}{\partial y}\right|_{K}$ and $x_{3}=\left.\left(\frac{\partial}{\partial x}+\frac{\partial}{\partial y}\right)\right|_{K}$. It is cesy to seo that the rootor fielda $x_{1}, x_{2}$ and $x_{3}$ are linearly Independent over $C$ only at the point $(0,0), 1,0 . \Delta_{c}\left(x_{1}, x_{2}, x_{3}\right)=$ $=\{(0,0)\}$. Marpover, wo have $\Delta_{\text {reg }-c}\left(x_{1}, x_{2}, x_{3}\right)=\Delta\left(x_{1}, x_{2}, x_{3}\right)=\emptyset$.

B I a m 1 . 1.7. Consider the vector riolda $x_{1}, x_{2} \in X\left(B^{2}\right)$ defined as follows: $x_{1}=\gamma \cdot \pi_{1} \frac{\partial}{\partial x}$ and $x_{2}=$ $=\gamma \cdot \pi_{2} \frac{\partial}{\partial y}$, where $\gamma: B \rightarrow B$ is a smooth map of the form

$$
t=\left\{\begin{array}{lll}
0 & \text { for } t \leqslant 0 \\
-\frac{1}{t} & \text { for } t>0
\end{array}\right.
$$

and $\pi_{1}, \pi_{2}$ are the projeotions of $\mathrm{B}^{2}$ onto the first and seoond coordinate, respectively. We observe that $\Delta_{c}\left(x_{1}, x_{2}\right)=$ $=\left\{(x, y) \in B^{2}, x \geqslant 0\right.$ and $\left.s \geqslant 0\right\}$ and $\Delta_{\text {reg }-C}\left(x_{1}, x_{2}\right)=\Delta\left(x_{1}, x_{2}\right)=$ $=\left\{(x, y) \in B^{2}: x>0\right.$ and $\left.y>0\right\}$.

By an eapg verifloation wo get

P r O p 081 t 10 n 1.8. For vector flelds $x_{1}, \ldots, x_{k} \in X_{f}(H, N)$, the set $\Delta_{C}\left(x_{1}, \ldots, x_{k}\right)$ is closed in $y$ and the sets $\Delta_{\text {reg }-C}\left(x_{1}, \ldots, x_{k}\right)$ and $\Delta\left(x_{1}, \ldots, x_{k}\right)$ are open in $M$.

2. Relative C-Iinear Independence

Do I I n 1 t 1 o $n$ 2.1. Veotor f1elds $x_{1}, \ldots, x_{k} \epsilon$ $\in \mathfrak{X}_{f}(M, N)$ are seid to be relatively C-linearls 1ndependent on $A$ if $A \subset M$ and the vector fielda $x_{1 \mid A} \ldots, x_{k \mid A} \in X_{f_{\mid A}}(A, N)$ are $C_{A}$-linearls independent.

The family of all subsets A of 1 such that the vector fields $X_{1}, \ldots, X_{k}$ are relatively C-linearly independent on $A$ will be denoted by $\operatorname{Re}_{c}\left(x_{1}, \ldots, x_{k}\right)$. 
Using the fact that the differential epace $M$ is a C-regular topological space, one can easily prove

I a m a 2.2. If $x_{1}, \ldots, x_{k} \in \mathfrak{X}_{f}(M, N)$, then the veotor flelde $x_{1}, \ldots, x_{k}$ are relatively C-linearly independent on $A \subset M$ if and only if, for anj $\alpha^{1}, \ldots, \alpha^{k} \in C$, the condition $\alpha_{\mid A}^{1} X_{1 \mid A}+\ldots+\alpha_{\mid A}^{k} X_{k \mid A}=0$ implies the identities $\alpha_{\mid A}^{1}=$ $=\ldots=\alpha_{\left.\right|_{A}}^{k}=0$.

$R$ m a r 2.3. From Definttions 1.1 and 2.1 it followe that if the vector fields $X_{1}, \ldots, I_{k} \in X_{p}(M, N)$ are relatively C-linearly independent on $A$, then $A \subset \Delta_{C}\left(x_{1}, \ldots, x_{k}\right)$. The converse 1s not true because if we take, for instance, Bxample 1.7, we have $B=\left\{(x, y) \in B^{2}: x \geqslant 0\right.$ and $\left.z=0\right\} \subset \Delta_{C}\left(x_{1}, x_{2}\right)$ but the vector fields $x_{1}, \ldots, x_{k}$ are not relatively C-linearly independent on $B$.

P I O P O 1 t 1 on 2.4. For any vector flelds $x_{1}, \ldots, x_{k} \in X_{f}(u, N)$, the family Re ${ }_{C}\left(x_{1}, \ldots, x_{k}\right)$ has the followIng properties:

(1) if $\left\{A_{t}\right\}_{t \in T} \operatorname{ReI_{C}}\left(x_{1}, \ldots, x_{k}\right)$, then $\bigcup_{t \in T} A_{t} \in \operatorname{Re} I_{C}\left(x_{1}, \ldots, x_{k}\right)$

(2) if $A \in \operatorname{ReI}_{C}\left(x_{1}, \ldots, x_{k}\right)$, then ol $A \in \operatorname{ReI}_{C}\left(x_{1}, \ldots, x_{k}\right)$

(3) If $A \in R \theta I_{C}\left(X_{1}, \ldots, X_{K}\right)$ and $U$ is an open subset of $M$ such that $A \cap U \neq \varnothing$, then $A \cap U \in \operatorname{Re}_{C}\left(x_{1}, \ldots, x_{k}\right)$

(4) if $A \in \operatorname{Re} I_{C}\left(x_{1}, \ldots, X_{k}\right)$ and $B \subset A \subset C I B$, then $B \in \operatorname{Re} I_{C}\left(x_{1}, \ldots, x_{k}\right)$

Proof. (1). Let $A=\bigcup_{t \in T} A_{t}$ and let $\alpha^{1}, \ldots, \alpha^{k} \in C_{A}$ be functions such that condition $\alpha^{1} X_{1 \mid A}+\ldots+\alpha^{k} x_{k \mid A}=0$ 1s fulfilled. Hence the conditions $\alpha_{\mid A_{t}}^{1} x_{1 \mid A_{t}}+\ldots+\alpha_{\mid A_{t}}^{k} X_{k \mid A_{t}}=0$ are fulfilled for ang $t \in T$ and since $A_{t} \in \operatorname{Re} I_{C}\left(x_{1}, \ldots, x_{k}\right)$, therefore $\alpha_{\mid A_{t}}^{1}=\ldots=\alpha_{\mid A_{t}}^{k}=0$. Thus, $a_{\left.\right|_{A}}^{1}=\ldots=a_{\left.\right|_{A}}^{k}=0$ and conseguently, $A \in \operatorname{ReI}_{C}\left(x_{1}, \ldots, x_{k}\right)$. 
(2) Let $B=c I A$, and let $\alpha^{1}, \ldots, \alpha^{k} \in C_{B}$ be functions suoh that $\alpha^{9} X_{1 \mid B}+\ldots+\alpha^{k} X_{k \mid B}=0$. In particular, we have $\alpha_{\mid A}^{1} x_{1 \mid A}+\ldots+\alpha_{\mid A}^{k} x_{k \mid A}=0$ and since $A \in \operatorname{Rel}_{C}\left(x_{1}, \ldots, x_{k}\right)$, so $\alpha_{\left.\right|_{A}}^{1}=\ldots=\left.\alpha\right|_{A} ^{k}=0$. Hence we obtain $\alpha^{1}=\ldots=\alpha^{k}=0$ and, therefore, $B \in \operatorname{Re}_{C}\left(x_{1}, \ldots, x_{k}\right)$.

(3) Let $\beta^{1}, \ldots, \beta^{k} \in C$ be functions such that $\beta_{\mid B}^{1} x_{1 \mid B}+$ $+\ldots+\beta_{\mid B}^{k} X_{k \mid B}=0$ where $B=A \cap U$, and let $p_{0} \in B$. There existe a function $\lambda \in C$ such that $\lambda\left(p_{0}\right)=1$ and $\lambda(q)=0$ for anj $g \in \mathbb{M} U$. Put $\alpha^{1}=\lambda \beta^{1}, 1=1,2, \ldots, k$ and note that $\alpha_{\mid A}^{1} \mathbb{X}_{1 \mid A}+$ $+\ldots \alpha_{\mid A}^{k} x_{k \mid A}=0$. Since $A \in \operatorname{Re} I_{C}\left(x_{1}, \ldots, x_{k}\right)$, therefore $\alpha_{\mid A}^{1}=\ldots=\alpha_{\mid A}^{k}=0$. In particular, $\beta^{1}\left(p_{0}\right)=\lambda\left(p_{0}\right) \beta^{1}\left(p_{0}\right)=$ $=\alpha^{1}\left(p_{0}\right)=0,1=1,2, \ldots, k_{0}$ and conseguentzy, $\beta_{\left.\right|_{B}}^{1}=\ldots=$ $=\left.\beta\right|_{B} ^{k}=0$ beoause $P_{0} \in B$ is an arbitrery point of $B_{0}$. Henoo, by Lemma 2.2, we get $B \in \operatorname{Re}_{C}\left(x_{1}, \ldots, x_{k}\right)$.

(4) Let $\beta^{1}, \ldots, \beta^{k} \in C$ be functions satisfying the condition $\beta_{\mid B}^{1} x_{1 \mid B}+\ldots+\beta_{\mid B}^{k} x_{k \mid B}=0$, and let $x_{0}=\beta^{1} x_{1}+\ldots+\beta^{k} x_{k}$. By Proposition 1.8, the set $\Psi \backslash \Delta\left(x_{0}\right)$ is closed and, sinoe

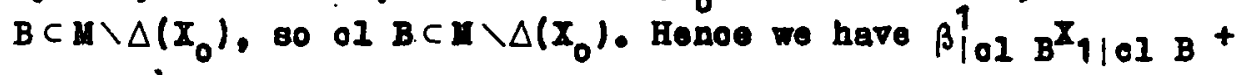
$\ldots+\beta_{\mid 01}^{k} \mathbf{B}_{\mathbf{z} \mid 01 \mathrm{~B}}=\mathbf{X}_{0 \mid C 1 \mathrm{~B}}=0$. In partioular, Binoe A CcI B, wo obtain $\beta_{\mid A}^{1} X_{1 \mid A}+\ldots+\beta_{\mid A}^{k} X_{k \mid A}=0$ and, s1noo $\Delta \in \operatorname{Rel}_{C}\left(x_{1}, \ldots, x_{k}\right)$, therefore $\beta_{\left.\right|_{A}}^{1}=\ldots=\beta_{A}^{k}=0$. Thus, $\beta_{\mid B}^{1}=\ldots=\beta_{\mid B}^{k}=0$, and, by Lemme 2.2, we get $B \in \operatorname{Re} I_{C}\left(x_{1}, \ldots, x_{k}\right)$.

De $f 1 \mathrm{n} 1+1$ on 2.5. For any $x_{1}, \ldots, x_{k} \in X_{f}(M, N)$, we adopt the following notation

$$
\Delta_{r \theta 1}-c^{\left(x_{1}, \ldots, x_{k}\right)}=\bigcup\left\{A \subset M, A \in R_{\theta}{ }_{c}\left(x_{1}, \ldots, x_{k}\right)\right\} \bullet
$$

From Definitions 1.2, 2.5 and Proposition 2.4 wo have

C O I 011 a I g 2.6. If $x_{1} \ldots, x_{k} \in x_{P}(x, I)$, then $\Delta_{\text {reg }}-c\left(x_{1} \ldots \ldots, X_{k}\right)$ is the ereatest open set of $\operatorname{Re} I_{c}\left(X_{1}, \ldots, x_{k}\right)$ 
and $\Delta_{\text {rel }}-c^{\left(X_{1}, \ldots, X_{k}\right)}$ is the greatest closed set of $\operatorname{ReI}_{C}\left(X_{1} \ldots, X_{k}\right)$.

Next, from (3) of Proposition 2.4 we get

$C$ or $\circ 11$ a $y$ 2.7. If $A \in R_{\theta} I_{C}\left(x_{1}, \ldots, x_{k}\right)$ and $U \subset A$ is an open nonempty set, then $U \in \operatorname{Re} I_{C}\left(x_{1}, \ldots, X_{k}\right)$.

C o r 011 a $\mathrm{y}$ 2.8. An open set $U \subset M$ belongs to $R I_{C}\left(X_{1}, \ldots, X_{k}\right)$ if and only if $U$ is nonempty and

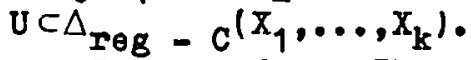

P r 0 o $f$ The nocessity results from Corollary 2.6, wheress the sufficiency - from 2.6 and 2.7.

C o r 011 a r 7 2.9. If $A \in \operatorname{ReI}_{C}\left(x_{1}, \ldots, X_{k}\right)$ and $B C A$ Is a set such that ol $B=c I A$, then $B \in \operatorname{Re} I_{C}\left(X_{1}, \ldots, X_{k}\right)$.

Pro of. From (2) of Proposition 2.4 we got that cl $A \in \operatorname{Rel} C\left(X_{1}, \ldots, X_{k}\right)$. Since $B C$ ol $A C 01 B$, therefore by (4) of Proposition 2.4, we have $B \in \operatorname{Re} I_{C}\left(x_{1}, \ldots, x_{k}\right)$.

\section{Connections between different notions of Iinear} Inde pendence

I e m m a 3.1. Let $Y, X_{1}, \ldots, X_{k} \in X_{f}\left(H_{0} N\right)$. If $\Delta\left(X_{1}, \ldots, X_{k}\right)=M$ and $\Delta\left(Y, X_{1}, \ldots, X_{k}\right)=0$, then there exiets exaotly one sequenoe of functions $\alpha^{1}, \ldots, \alpha^{k} \in C$, suoh that $Y=\alpha^{1} X_{1}+\ldots+\alpha^{k} X_{k}$.

$P r \circ 0 f$. By assumption, for any $p \in M$, the vootors $x_{1}(p), \ldots, X_{k}(p)$ are linearly independent but the vectore $Y(p), X_{1}(p), \ldots, X_{k}(p)$ are linearly dependent. Therefore, there existe exaotly one eequence $\alpha^{1}, \ldots, \alpha^{k}$ of real functions, ench that

$$
Y(p)=\alpha^{1}(p) X_{1}(p)+\ldots+\alpha^{k}(p) X_{k}(p) \quad p \in w_{0}
$$

We shall show that $\alpha^{1}, \ldots, \alpha^{k} \in C$, Really, let $p_{0} \in M$, and note that there exists eunctions $\beta^{1}, \ldots, \beta^{k} \in D$ ouch that $\operatorname{det}\left(x_{1}\left(p_{0}\right)\left(\beta^{j}\right)\right) \neq 0$. Since the veotor flelds $\Sigma_{1} \ldots, I_{k}$ are smooth, there exists an open neighbomhood $U$ of $p_{0}$ suoh that $\operatorname{det}\left(x_{1}(p)\left(\beta^{j}\right)\right) \neq 0$ for $p \in U$. 
Consider the following system of linear equations

$$
\alpha^{1}(p) x_{1}(p)\left(\beta^{1}\right)+\ldots+\alpha^{k}(p) X_{k}(p)\left(\beta^{1}\right)=Y(p)\left(\beta^{1}\right)
$$

for $1=1, \ldots, k$, where $p \in U$. Since the function $p \rightarrow \operatorname{det}\left(X_{1}(p)\left(\beta^{j}\right)\right) \neq 0$ is smooth on $U$, the sjstem of Iinear equations has exactiy one smooth solution, 1.e. $\alpha_{\mid U}^{1} \ldots \alpha_{\mid U}^{k} \in C_{U}$. Thus, since $C$ is olosed with respect to the localization, we get $\alpha^{1}, \ldots, \alpha^{k} \in C$.

I a in a 3.2. If $X_{1}, \ldots, X_{k} \in X_{p}(U, X)$ and $U$ is an open subset of $M$, then $\Delta_{C_{0}}\left(x_{1 \mid \sigma}, \ldots, x_{k \mid U}\right)=\Delta_{C}\left(x_{1}, \ldots, x_{k}\right) \cap U$.

$P r \circ 0 f$ Let $p \in \Delta_{C_{U}}\left(x_{1 \mid U}, \ldots, x_{k \mid U}\right)$, and let $\alpha^{1}, \ldots, \alpha^{k} \in C$ be funotions such that $\alpha^{1} x_{1}+\ldots+\alpha^{k} x_{k}=0$. Henoe $\alpha\}_{U}^{1} x_{1 \mid U}+\ldots+\alpha_{\mid U}^{k} x_{k \mid U}=0$ and, eince $p \in \Delta_{C_{U}}\left(x_{1 \mid U}, \ldots\right.$ $\left.\ldots, x_{k \mid U}\right)$, so $\alpha^{1}(p)=\ldots=\alpha^{k}(p)=0$. Consequently, $\Delta_{c_{v}}\left(x_{1 \mid U}, \ldots, x_{k \mid U}\right) \subset \Delta_{c}\left(x_{1}, \ldots, x_{k}\right) \cap 0$.

Conversels, lot $p_{0} \in \Delta_{C}\left(x_{1}, \ldots, x_{k}\right) \cap U$, and lot $\beta^{1}, \ldots, \beta^{k} \in C_{U}$ be functions such that $\beta^{1} x_{1 \mid}+\ldots+\beta^{k} x_{k \mid U}=0$. Then there existe an open neighbourhood $U_{0} \subset \delta$ of $p_{0}$ and functions $\gamma^{1}, \ldots, \gamma^{k} \in C$, such that for $1=1,2, \ldots, k, \beta_{\mid U}^{1}=\gamma_{\mid U}^{1}$. Since differential space in is a C-rogular topological spaos, there exists a function $\lambda \in C$ such that $\lambda\left(p_{0}\right)=1$ and $\lambda(q)=0$ for ang $q \in M \backslash \delta_{0}$. Put $\alpha^{1}=\lambda \gamma^{1}, 1=1,2, \ldots, k$ and note that the condition $\beta^{1} x_{1 \mid U}+\ldots+\beta^{k} x_{k \mid U}=0$ implieg the identity $\alpha^{1} x_{1}+\ldots+\alpha^{k} x_{k}=0$. Since $p_{0} \in \Delta_{c}\left(x_{1}, \ldots, x_{k}\right)$, it follows that $\alpha^{1}\left(p_{0}\right)=\ldots=\alpha^{k}\left(p_{0}\right)=0$.

Henoo $\beta^{1}\left(p_{0}\right)=\gamma^{1}\left(p_{0}\right)=\lambda\left(p_{0}\right) \alpha^{1}\left(p_{0}\right)=0,1=1,2, \ldots, k$, and, conseguentlJ $\Delta_{C}\left(x_{1}, \ldots, x_{k}\right) \cap U \subset \Delta_{C_{0}}\left(x_{1 \mid U}, \ldots, x_{k \mid 0}\right)$.

I o m m a 3.3. If $x_{1}, \ldots, x_{k} \in X_{p}(y, I)$ and $u \in \operatorname{ReI} I_{c}\left(x_{1}, \ldots, x_{k}\right)$, then $\Delta\left(x_{1}, \ldots, x_{k}\right) \neq \emptyset$. 
P r 0 O $f$. Wo shall prove this by induotion on $k$. of course, the condition is fulfilled for $k=1$. Assume that it is fulfilled for $k=n$ and suppose that it is not for $k=n+1$. Then there exist vector fields $x_{1}, \ldots, x_{n+1}$ such that $M \in R \in I_{C}\left(x_{1}, \ldots, x_{n+1}\right)$ and $\Delta\left(x_{1}, \ldots, x_{n+1}\right)=\varnothing$. It is eagy to see that $M \in \operatorname{Re} I_{C}\left(x_{1}, \ldots, X_{n}\right)$ and, by our assumption, we have $\Delta\left(x_{1}, \ldots, x_{n}\right) \neq \varnothing$. Let $U=\Delta\left(x_{1}, \ldots, x_{n}\right)$, and note that $U$ is an open set in $M$ (see 1.8). On the other hand, we have $\Delta\left(x_{1 \mid U}, \ldots, x_{n \mid U}\right)=U$ and $\Delta\left(x_{1 \mid U}, \ldots, x_{n+1 \mid U}\right)=\varnothing$. Thus, from Lemma 3.1 we conclude that there exist functions $\alpha^{1}, \ldots, \alpha^{n} \in C_{U}$ such that $x_{n+1 \mid U}=\alpha^{1} x_{1 / U}+\ldots+\alpha^{n} x_{n \mid U}$. This is a contradiotion because, by Corollary 2.7, we have $U \in \operatorname{Re} I_{C}\left(x_{1}, \ldots, x_{n+1}\right)$.

P I O p O a it $10 \mathrm{n}$ 3.4. If $x_{1}, \ldots, x_{k} \in \mathscr{X}_{f}(\mathbb{M}, N)$ then the following relations are satisfied:

(a) $\Delta\left(x_{1}, \ldots, x_{k}\right) c \Delta_{\text {reg }-c}\left(x_{1}, \ldots, x_{k}\right) c$

$$
c \Delta_{r \in 1-c}\left(x_{1}, \ldots, x_{k}\right) \subset \Delta_{c}\left(x_{1}, \ldots, x_{k}\right) \text {, }
$$

(b) $\operatorname{cl} \Delta\left(x_{1}, \ldots, x_{k}\right)=c 1 \Delta_{r e g-c}\left(x_{1}, \ldots, x_{k}\right)=\Delta_{r e 1-c}\left(x_{1}, \ldots, x_{k}\right)_{1}$

(0) $\Delta_{\text {reg }-C}\left(x_{1}, \ldots, x_{k}\right)=$ int $\Delta_{r \in I-c}\left(x_{1}, \ldots, x_{k}\right)=$

$=$ int $\Delta_{C}\left(x_{1}, \ldots, x_{k}\right)$.

P r O O P. (a) Observe that $\Delta\left(x_{1}, \ldots, x_{k}\right)=$ $=\left\{p \in \mathbb{M}:\{p\} \in \operatorname{Re} I_{C}\left(x_{1}, \ldots, x_{k}\right)\right\}$. Hence, from (1) of Proposition 2.4 we have $\Delta\left(x_{1}, \ldots, x_{k}\right) \in \operatorname{Re}_{c}\left(x_{1}, \ldots, x_{k}\right)$. Since the set $\Delta\left(X_{1}, \ldots, X_{k}\right)$ is open, by Proposition 1.8 , so from Example 1.2 we conclude that $\Delta\left(x_{1}, \ldots, x_{k}\right) \subset \Delta_{\text {reg }-C}\left(x_{1}, \ldots, x_{k}\right)$. The oonolueion

$$
\operatorname{reg-C}\left(x_{1}, \ldots, x_{k}\right) \subset \Delta_{r e l-C}\left(x_{1}, \ldots, x_{k}\right)
$$

results from Defintion 2.5 and Corollary 2.6. To prove inclusion $\Delta_{\text {rel-c }}\left(x_{1}, \ldots, x_{k}\right) \subset \Delta_{c}\left(x_{1}, \ldots, X_{k}\right)$, consider an arbitrary point $p \in \Delta_{r \in 1-C}\left(x_{1}, \ldots, x_{k}\right)$. Let $\alpha^{1}, \ldots, \alpha^{k} \in C$ be funotions such that $\alpha^{1} x_{1}+\ldots+\alpha^{k} x_{k}=0$. Hence $\alpha_{\mid A}^{1} x_{1 \mid A}+\ldots$ $\ldots+\alpha_{\mid A}^{k} x_{k \mid A}=0$, where $A=\Delta_{r e l-C}\left(x_{1}, \ldots, x_{k}\right)$, and sinoe 
$A \in \operatorname{Re} I_{C}\left(x_{1}, \ldots, x_{k}\right)$ (eoe Proposition 2.4), therefore $a_{1 A}^{1}=$ $\ldots=a_{1}^{k}=0$. In particular, wo have $\alpha^{1}(p)=\ldots=\alpha^{k}(p)=0$, which moans that $p \in \Delta_{c}\left(x_{1}, \ldots, x_{k}\right)$.

(b) From $(a)$ and eince the set $\Delta_{\text {rel-C }}\left(X_{1}, \ldots, X_{k}\right)$ is closed (see Proposition 1.8) we get the oonclusions: $c 1 \Delta\left(x_{1}, \ldots, x_{k}\right) \subset$ col $\Delta_{\text {reg }-C}\left(x_{1}, \ldots, x_{k}\right) \subset \Delta_{\text {rel-C }}\left(x_{1}, \ldots, x_{k}\right)$. So it suffices to prove the oonolusion $\Delta_{r e l-c}\left(x_{1}, \ldots, x_{k}\right)<01 \Delta\left(x_{1}, \ldots, x_{k}\right)$. Suppose that this inclusion is no true. Then there extets an open set $U \subset M$ such that $U \cap \Delta_{x \in I-C}\left(x_{1}, \ldots, x_{k}\right) \neq \emptyset$ and $U \cap \Delta\left(x_{1}, \ldots, X_{k}\right)=\emptyset$. Let $A=U n \Delta_{\text {rel-c }}\left(x_{1}, \ldots, x_{k}\right)$. Observe that $A \in \operatorname{Rel}_{C}\left(x_{1}, \ldots, x_{k}\right)$ (see Corollary 2.6 and $(3)$ of Proposition 2.4). Hence it results that $A \in \operatorname{Rel}_{C_{A}}\left(X_{1 \mid A}, \ldots, X_{k \mid A}\right)$ as well as $\Delta\left(X_{1 \mid A}, \ldots, X_{k \mid A}\right)=\varnothing$. But this is a contradiction because, by Lemma 3.3 , we have $\Delta\left(x_{1 \mid A}, \ldots, x_{k \mid A}\right) \neq \varnothing$. Therefore the last incluston is true.

(c) Since the set $\Delta_{\text {reg-C }}\left(X_{1}, \ldots, X_{k}\right.$ ) is open (see Proposition 1.8) so from (a) we obtain the conclusions:

$$
\Delta_{\text {reg }-C}\left(x_{1}, \ldots, x_{k}\right) \subset \text { int } \Delta_{r \theta 1-C}\left(x_{1}, \ldots, x_{k}\right) c \text { int } \Delta_{C}\left(x_{1}, \ldots, x_{k}\right) .
$$

Thus, it is enough to show the inclusion int $\Delta_{c}\left(x_{1}, \ldots, x_{k}\right) c$ $c \Delta_{\text {reg }-C}\left(x_{1}, \ldots, x_{k}\right)$. Indeed, let $U=$ int $\Delta_{C}\left(x_{1}, \ldots, X_{k}\right)$ and observe that $\Delta_{C_{0}}\left(x_{1 \mid U}, \ldots, x_{k \mid \sigma}\right)=\Delta_{C}\left(x_{1}, \ldots, x_{k}\right) \cap U=U$ (seo Lemma 3.2). From this we conclude that $U \in \operatorname{R\theta } I_{C}\left(x_{1}, \ldots, x_{k}\right)$ and we get UC $\Delta_{\text {reg }-C}\left(x_{1}, \ldots, X_{k}\right)$ (see Corollarg 2.6). Therefore the last inclusion take place, which completes the proof of the theorem.

From Proposition 3.4 we immediately obtain

C Or O I I a $g$ 3.5. If $x_{1}, \ldots, x_{k} \in X_{f}(M, N)$ then the following conditions are equivalent:

$$
\begin{gathered}
\Delta_{C}\left(x_{1}, \ldots, x_{k}\right)=M \\
\Delta_{r \in 1-C}\left(x_{1}, \ldots, x_{k}\right)=M
\end{gathered}
$$




$$
\begin{gathered}
\Delta_{r \theta g-C}\left(x_{1}, \ldots, x_{k}\right)=x \\
\operatorname{cl} \Delta\left(x_{1}, \ldots, x_{k}\right)=u_{0}
\end{gathered}
$$

Of oourse, each of these equivalent conditions means that

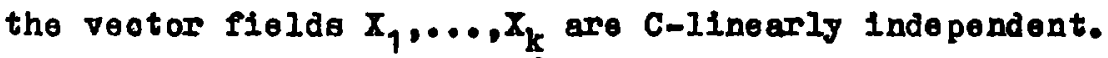
C O I 011 a I $\mathrm{g}$ 3.6. Veotor flelda $x_{1}, \ldots, x_{k} \in$ $\in X_{P}(M, N)$ are C-linearly independent if and only $1 f$ there exists an open and denee set $u_{0} \subset M$ such that, for ang point $p \in u_{0}, x_{1}(p) \wedge \ldots \wedge x_{k}(p) \neq 0$.

P $r 001$. If the veotor fields $I_{1}, \ldots, I_{k}$ are C-linearly Independent then we mes put $M_{0}=\Delta\left(x_{1}, \ldots, x_{k}\right)$ (aee (4) of Corollary (3.5). Conversely, we observe that if $u_{0}$ satiafies the conditions of this Corollary then $x_{0} c \Delta\left(x_{1}, \ldots, x_{k}\right)$, so (4) of Corollary 3.5 holda.

As an another formulation of Corollary 3.6 we get

C Or 011 a I 8 3.7. If the vector flelds $x_{1}, \ldots, x_{k} \epsilon$ $\in \mathfrak{X}_{f}(\mathrm{M}, \mathbb{N})$ generate the C-module $x$, then $x_{1}, \ldots, \mathrm{x}_{\mathrm{k}}$ form the C-basis of $X$ if and only if there exista an open and deneo set $M_{0} \subset M$ such that the $C_{M_{0}}$-module $X_{M_{0}}$ is an n-dimensional differential module (see [1]).

This Corollary Impliss

C o r o I I a $y$ 3.8. If $X$ is a locally freo C-submodule of $X_{f}(M, N)$ then there exists an open and dener set $M_{0} \subset M$ such that, the $C_{u_{0}}$-module 1e a differential module.

R a m a $r$ 3.9. C-submodule $X$ of $\mathfrak{X}_{f}(\mathrm{H}, \mathrm{N})$ is said to be a locally free if, for any point $p \in M$, there exist an open neighbourhood $U$ of $p$ and vector fields $x_{1}, \ldots, x_{k} \in X_{U}$ which form the $C_{U}$-basis of $x_{U}$. Note that if $K$ is the differential apace defined in example $1.6, X=X(K)$ and $\mathrm{K}_{0}=$ $=\mathrm{K} \backslash\{(0,0)\}$ then $x_{\mathrm{K}_{0}}$ is a 1-dimensional differential module but $X$ is not a locally free module. 


\section{REFEREYCES}

[1] R. S 1 k or k 1: Differential modules, Collog. Math., 24 (1971) 45-70.

[2] R. S 1 k O I B 1 : Introduotion to Differential Goometry. Wargzawa 1972 (In Polish).

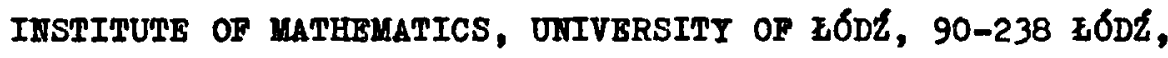
POLAIID;

INSTITUTE OF MATHEMATICS, TECHNICAL UNIVERSITY OF WARSAT, 00-661 TARSZATA

Reoolved February 7, 1983. 
